\title{
A Density-Based Ramp Metering Model Considering Multilane Context in Urban Expressways
}

\author{
Li Tang, ${ }^{1,2}$ Xia Luo, ${ }^{2,3}$ Pengfei Zhai, ${ }^{3}$ and Xunfei Gao ${ }^{3}$ \\ ${ }^{1}$ School of Automobile and Transportation, Xihua University, No. 999 Jinzhou Road, Chengdu, Sichuan 610039, China \\ ${ }^{2}$ Jiangsu Province Collaborative Innovation Center of Modern Urban Traffic Technologies, Sipailou No. 2, Nanjing 210096, China \\ ${ }^{3}$ School of Transportation and Logistics, Southwest Jiaotong University, No. 111 Second Ring Road, North Section 1, \\ Chengdu, Sichuan 610031, China
}

Correspondence should be addressed to Li Tang; tanglitraffic@gmail.com

Received 27 October 2016; Accepted 17 January 2017; Published 2 March 2017

Academic Editor: Gennaro N. Bifulco

Copyright (C) 2017 Li Tang et al. This is an open access article distributed under the Creative Commons Attribution License, which permits unrestricted use, distribution, and reproduction in any medium, provided the original work is properly cited.

\begin{abstract}
As one of the most effective intelligent transportation strategies, ramp metering is regularly discussed and applied all over the world. The classic ramp metering algorithm ALINEA dominates in practical applications due to its advantages in stabilizing traffic flow at a high throughput level. Although ALINEA chooses the traffic occupancy as the optimization parameter, the classic traffic flow variables (density, traffic volume, and travel speed) may be easier obtained and understood by operators in practice. This paper presents a density-based ramp metering model for multilane context (MDB-RM) on urban expressways. The field data of traffic flow parameters is collected in Chengdu, China. A dynamic density model for multilane condition is developed. An error function represented by multilane dynamic density is introduced to adjust the different usage between lanes. By minimizing the error function, the density of mainstream traffic can stabilize at the set value, while realizing the maximum decrease of on-ramp queues. Also, VISSIM Component Object Model of Application Programming Interface is used for comparison of the MDB-RM model with a noncontrol, ALINEA, and density-based model, respectively. The simulation results indicate that the MDB-RM model is capable of achieving a comprehensive optimal result from both sides of the mainstream and on-ramp.
\end{abstract}

\section{Introduction}

Building expressways or elevated expressways inside the city to relieve severe congestion and its related problems is a popular strategy in many metropolitan areas of the world. However, as urban planners must contend with limited land resources, increasing capacity simply by adding infrastructure is not sustainable. Thus, Intelligent Transportation System (ITS) is introduced as an alternative approach to tackle the issue of traffic jams [1]. Although many efforts have been done to optimize traffic operations using ITS techniques, the topic of motorway traffic regulation is still an open field for researchers and for practical implementation [2]. On-ramp metering is regarded as one of the most effective traffic control strategies to exploit the potential of supply in expressways [3]. Through the use of traffic lights, local ramp metering corresponds to controlling the traffic flow merging into the mainstream from the on-ramp, so as to maximize the mainstream flow downstream of the on-ramp in response to prevailing traffic conditions $[4,5]$. Since it was first introduced on I-290 in Chicago in 1963, on-ramp metering has been implemented on major expressways in many big cities around the world. Examples include Route 101 in Los Angeles [6], Boulevard Périphérique in Paris [7, 8], and the A10 West Motorway in Amsterdam [9]. Coordinated ramp metering strategies make use of measurements from an entire region of the network to control all metered ramps. Coordinated ramp metering approaches include multivariable control strategies [10], optimal control strategies [11], and further heuristic algorithms [12]. A few coordinated ramp metering strategies have been deployed recently in the field, such as the I25 Motorway in Denver [13] and the M1/M3 Motorway in Queensland [14]. Another extensive control method is ramp integration control metering, which combines ramp 
metering and other traffic control strategies, such as variable speed limits and route guidance, so as to ameliorate traffic performance both in motorways and in urban roads. During the last decades, ramp integration control metering has been well developed. The field tests are also well performed [15].

The current employed ramp metering algorithms largely use ALINEA, the first local ramp metering control strategy based on straightforward application of classical feedback control theory, so as to achieve and maintain maximum capacity occupancy [16]. Taking advantage of the feedback mechanism, ALINEA displays superior performance compared with feedforward-based strategies (e.g., demandcapacity and occupancy strategies) in terms of smoothly reacting to slight differences in occupancy and stabilizing traffic flow at a high throughput level $[17,18]$. ALINEA is developed upon traffic occupancies, the measurements of which may not be readily related to the classic traffic flow variables. Thus, a great number of efforts have been made to improve ALINEA, like GA-based ALINEA in 2002 [19, 20], UP-ALINEA, FL-ALINEA, and UF-ALINEA in 2003 [21], AD-ALINEA and AU-ALINEA in 2004 [22], PI-ALINEA in 2007 [23, 24], IFT-tuned ALINEA in 2010 [25, 26], and so forth. In most of the improved algorithms, traffic volume is introduced as the set value in order to operate more conveniently in practical applications. However, traffic flow does not uniquely characterize the traffic state (i.e., the same values of traffic volume may indicate both light and congested traffic), as well as travel speed. As a result, a stream of scholars suggested using density as the key objective of optimizing ramp metering rates [27]. Generally speaking, this will bring about benefits in two main aspects. First, the density of the mainstream traffic flow can stabilize at the expected value, like ALINEA. Meanwhile, the queue length in the on-ramp can be minimized as much as possible. Second and more significant, compared with occupancy data, the obtaining of which is relied on for the layout of underground loops, the measurement of density is much easier and more convenient (e.g., using video recording or even unmanned aerial vehicles).

This paper focuses on modeling density-based ramp metering control for multilane expressways (MDB-RM) in urban areas. The following contributions have been made: a density-based ramp metering model considering multilane situations in real life is built. A dynamic density model for multilane condition is developed on the basis of METANET model. An error function represented by multilane dynamic density is introduced into the MDB-RM model to adjust the different usage among lanes and minimize objective for optimization. Real traffic data is collected from the Second Ring Elevated Expressway in Chengdu. It is used for parameter fixing and simulation. VISSIM 7 is chosen as the simulation platform running our proposed model and compared models.

\section{MDB-RM Model}

2.1. Dynamic Density Model for Multilane Condition. Since Lighthill and Whitham published their famous dynamic macroscopic traffic flow modeling paper [28], much work has been done to provide mathematical and computational approaches for describing the characteristics of traffic flow. In this paper, the dynamic density model is developed on the basis of the META [29, 30] and METANET [31, 32] models proposed by Messmer and his collaborators. Adopting and unifying the definitions used by Papageorgiou and Kotsialos (2002) [3], an expressway can be divided into $N$ segments of equal length $L$. The traffic density $\rho(k+1, i)$ for each lane in a segment $i$ at time $t=(k+1) T$ can be represented as

$$
\rho(k+1, i)=\rho(k, i)+\frac{T}{L \lambda}[q(k, i-1)-q(k, i)],
$$

where $k=1,2, \ldots, K$ is the discrete time index, $T$ is sample time, which is typically equal to $5 \cdots 15 \mathrm{~s}, q(k, i)$ is the number of vehicles leaving segment $i$ during the time period $[k T,(k+$ 1)T], divided by $T$, and $\lambda$ is the number of lanes.

Tan and Tang improved the META model in the aspect of considering lane change behavior and additional factors, such as safety space between vehicles, speed, and route choice. The density model in multilane situations reads

$$
\begin{aligned}
& \rho^{r s}(k+1, i, l)=\rho^{r s}(k, i, l)+\frac{T}{L}\left[q^{r s}(k, \varphi(i, l))\right. \\
& \left.-q^{r s}(k, i, l)+u^{r s}(k, i, l)-e^{r s}(k, i, l)\right] \\
& +\sum_{y \in C_{i}} n_{y l}^{r s}(k, i)-\sum_{y \in C_{i}} n_{l y}^{r s}(k, i),
\end{aligned}
$$

where $r$ is the origin of vehicle, $s$ is the destination of vehicle, $\varphi(i, l)$ is the set of lanes adjacent to lane $l$ in the upstream segment $i-1, \varphi(i, l)=\left\{(i-1 \cdot y):|1-y| \leq 1, y \in C_{i}\right\}, q^{r s}(k, i, l)$ is the number of vehicles leaving from lane $l$ of segment $i$ to downstream mainline during the time period $[k T,(k+1) T]$, divided by $T, u^{r s}(k, i, l)$ is the number of vehicles merging from on-ramp to lane $l$ of segment $i$ during the time period $[k T,(k+1) T]$, divided by $T, e^{r s}(k, i, l)$ is the number of vehicles leaving through off-ramp from lane $l$ of segment $i$ during the time period $[k T,(k+1) T]$, divided by $T$, and $n_{y l}^{r s}(k, i)$ is the number of vehicles changing from lane $y$ to lane $l$ of segment $i$ during the time period $[k T,(k+1) T]$.

Tan's model gives an adequate description of real traffic flow conditions on freeways. However, excessively detailed definitions of origin and destination substantially increase the difficulty of data acquisition, something which is not necessary in the ramp metering modeling. Thus, the density model is reformulated based on the following assumptions:

(i) The urban expressway is divided into $N$ segments of equal length $L$. The number of lanes in the expressway stays the same.

(ii) For segment $i$, there is at most one on-ramp or offramp connected with it.

(iii) Vehicles from the on-ramp can only merge into the outermost lane. Vehicles from the mainstream can only change to adjacent lanes. Cross-lane change is not allowed.

To simplify the model formula, a two-lane situation is first given. Define the inner lane as lane 1 and the outer lane as 


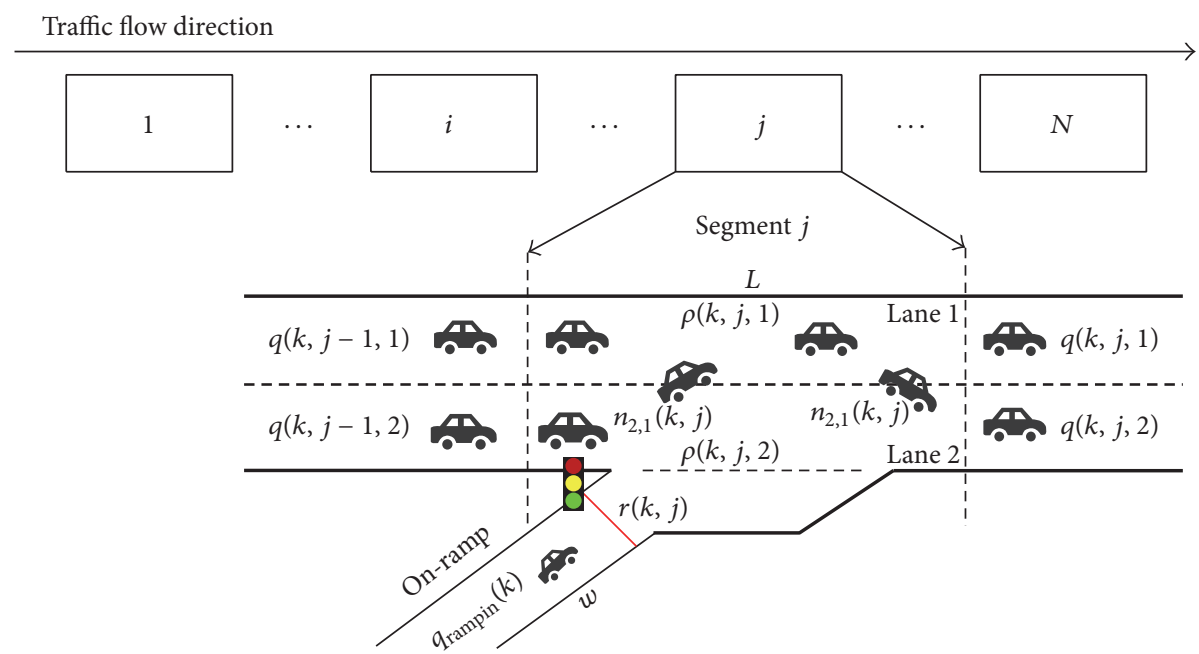

FIgURE 1: Layout of segment $i=j$ with an on-ramp.

lane 2 . When segment $i \neq j$, that means no on-ramp or offramp is connected. And when segment $i=j$, this means an on-ramp is connected. The layout of segment $j$ is shown as Figure 1 (since this paper focuses on the on-ramp metering control, only the on-ramp layout is given; the segment with an off-ramp can be deduced in the same process).

Thus, the dynamic density of inner lane and outer lane can be written as

$$
\begin{aligned}
& \rho(k+1, i, 1)=\rho(k, i, 1)+\frac{T}{L}[q(k, i-1,1)-q(k, i, 1) \\
& \left.+n_{2,1}(k, i)-n_{1,2}(k, i)\right], \\
& \rho(k+1, i, 2)=\rho(k, i, 2)+\frac{T}{L}[q(k, i-1,2)-q(k, i, 2) \\
& \left.+n_{1,2}(k, i)-n_{2,1}(k, i)\right] \quad i \neq j, \\
& \rho(k+1, i, 2)=\rho(k, i, 2)+\frac{T}{L}[q(k, i-1,2)-q(k, i, 2) \\
& \left.+u(k, i)+n_{1,2}(k, i)-n_{2,1}(k, i)\right] \quad i=j,
\end{aligned}
$$

where $q(k, i, 1)$ is the number of vehicles leaving from the inner lane of segment $i$ to downstream mainline during the time period $[k T,(k+1) T]$, divided by $T$, and $n_{1,2}(k, i)$ is the number of vehicles changing from inner lane to outer lane of segment $i$ during the time period $[k T,(k+1) T]$.

Define $\eta_{y, l}(k, i)$ as the ratio of the number of vehicles changing from lane $y$ to lane $l$ of segment $i$ to the number of vehicles in lane $y$ of segment $i$ during the time period $[k T,(k+1) T]$. Thus, (3) can be rewritten as

$$
\begin{aligned}
\rho(k+1, i, 1) & \\
= & \rho(k, i, 1)+\frac{T}{L}[q(k, i-1,1)-q(k, i, 1)] \\
& +\eta_{2,1}(k, i) \rho(k, i, 2)-\eta_{1,2}(k, i) \rho(k, i, 1),
\end{aligned}
$$

$$
\begin{aligned}
\rho(k+1, i, 2) \\
=\rho(k, i, 2)+\frac{T}{L}[q(k, i-1,2)-q(k, i, 2)] \\
\quad+\eta_{1,2}(k, i) \rho(k, i, 1)-\eta_{2,1}(k, i) \rho(k, i, 2)
\end{aligned}
$$$$
\text { if } i \neq j \text {, }
$$

$$
\begin{aligned}
\rho(k+ & 1, i, 2) \\
= & \rho(k, i, 2) \\
& +\frac{T}{L}[q(k, i-1,2)-q(k, i, 2)+u(k, j)] \\
& +\eta_{1,2}(k, i) \rho(k, i, 1)-\eta_{2,1}(k, i) \rho(k, i, 2)
\end{aligned}
$$$$
\text { if } i=j \text {. }
$$

And the dynamic density of on-ramp can be written as

$$
\begin{aligned}
\rho_{\text {ramp }}(k+1, i)= & \rho_{\text {ramp }}(k, i) \\
& +\frac{T}{w}\left[q_{\text {rampin }}(k)-r(k, i)\right],
\end{aligned}
$$

where $q_{\text {rampin }}(k)$ is the number of vehicles entering on-ramp during the time period $[k T,(k+1) T]$, divided by $T$, and $w$ is the length of on-ramp.

Furthermore, for multilane situations, define the total number of lanes as $X$. Let $l$ represent the $l$ th lane and $l=$ $1,2, \ldots, X$. When $l=1$, it represents the inner lane, and when $l=X$, it represents the outer lane. The multilane dynamic density model reads as follows. 
For inner lane,

$$
\begin{aligned}
\rho(k+1, i, l)= & \rho(k, i, l)+\frac{T}{L}[q(k, i-1, l)-q(k, i, l)] \\
& +\sum_{y \in C_{i}} \eta_{y, l}(k, i) \rho(k, i, y) \\
& -\sum_{y \in C_{i}} \eta_{l, y}(k, i) \rho(k, i, l)
\end{aligned}
$$

$$
\text { if } 1 \leq l \leq X-1
$$

For outer lane,

$$
\begin{aligned}
\rho(k+ & 1, i, X) \\
= & \rho(k, i, X)+\frac{T}{L}[q(k, i-1, X)-q(k, i, X)] \\
& +\sum_{y \in C_{i}} \eta_{y, X}(k, i) \rho(k, i, y) \\
& -\sum_{y \in C_{i}} \eta_{X, y}(k, i) \rho(k, i, X) \quad \text { if } i \neq j . \\
\rho(k+ & 1, i, X) \\
= & \rho(k, i, X) \\
& +\frac{T}{L}[q(k, i-1, X)-q(k, i, X)+u(k, i)] \\
& +\sum_{y \in C_{i}} \eta_{y, X}(k, i) \rho(k, i, y) \\
& -\sum_{y \in C_{i}} \eta_{X, y}(k, i) \rho(k, i, X) \quad \text { if } i=j .
\end{aligned}
$$

2.2. Density-Based Ramp Metering Model for Multilane Expressways. To build up the MDB-RM model, first we need to derive differential equations for the multilane dynamic density model. For segment $i$ without a connected on-ramp, that is, $i \neq j$, the variation of density for each lane is determined by outflow $q(k, i-1, l)$ from segment $i-1$ and $q(k, i, l)$ from segment $i$. Define $\Delta t=\Delta k \cdot T$; the variation of density when $i \neq j$ can be represented as

$$
\begin{array}{r}
\rho(k+\Delta k, i, l)=\rho(k, i, l)+\frac{\Delta t}{L}[q(k, i-1, l) \\
\left.-q(k, i, l)+\sum_{y \in C_{i}} n_{y, l}(k, i)-\sum_{y \in C_{i}} n_{l, y}(k, i)\right] .
\end{array}
$$

Transposing (8) and taking limit of both sides, we can get

$$
\begin{aligned}
& \lim _{\Delta t=0} \frac{\rho(k+\Delta k, i, l)-\rho(k, i, l)}{\Delta t} \\
& =\frac{q(k, i-1, l)-q(k, i, l)+\sum_{y \in C_{i}} n_{y, l}(k, i)-\sum_{y \in C_{i}} n_{l, y}(k, i)}{L} .
\end{aligned}
$$

Thus, the differential equation for segment $i \neq j$ reads

$$
\begin{gathered}
\dot{\rho}(k, i, l)=\frac{\mathrm{d} \rho(k, i, l)}{\mathrm{d} t}=\frac{1}{L}[q(k, i-1, l)-q(k, i, l) \\
\left.+\sum_{y \in C_{i}} n_{y, l}(k, i)-\sum_{y \in C_{i}} n_{l, y}(k, i)\right] .
\end{gathered}
$$

As for segment $i=j$, the differential equation stays the same with $i \neq j$ situation for inner lanes $1 \leq l \leq X-1$. And, for outer lane $l=X$, the variation of density is determined by the outflow $q(k, i-1, l)$ from segment $i-1, q(k, i, l)$ from segment $i$ and inflow $u(k, i)$ from on-ramp. Thus, the variation of density when $i=j$ can be represented as

$$
\begin{aligned}
& \rho(k+\Delta k, i, l)=\rho(k, i, l)+\frac{\Delta t}{L}[q(k, i-1, l) \\
& \left.-q(k, i, l)+r(k, i)+\sum_{y \in C_{i}} n_{y, l}(k, i)-\sum_{y \in C_{i}} n_{l, y}(k, i)\right] .
\end{aligned}
$$

Replicating the formula transformation like (9), the differential equation for segment $i=j$ reads

$$
\begin{aligned}
& \dot{\rho}(k, i, l)=\frac{\mathrm{d} \rho(k, i, l)}{\mathrm{d} t}=\frac{1}{L}[q(k, i-1, l)-q(k, i, l) \\
& \left.\quad+r(k, i)+\sum_{y \in C_{i}} n_{y, l}(k, i)-\sum_{y \in C_{i}} n_{l, y}(k, i)\right] .
\end{aligned}
$$

Thus, the differential equations of dynamic density considering lane change behavior in multilane expressways can be summarized as

$$
\begin{aligned}
& \dot{\rho}(k, i, l)=\frac{1}{L}\left[q(k, i-1, l)-q(k, i, l)+\sum_{y \in C_{i}} n_{y, l}(k, i)\right. \\
& \left.-\sum_{y \in C_{i}} n_{l, y}(k, i)\right] \quad \text { if } i \neq j \text { or } i=j, 1 \leq l \leq X-1, \\
& \dot{\rho}(k, i, l)=\frac{1}{L}[q(k, i-1, l)-q(k, i, l)+r(k, i) \\
& \left.+\sum_{y \in C_{i}} n_{y, l}(k, i)-\sum_{y \in C_{i}} n_{l, y}(k, i)\right] \quad \text { if } i=j, l=X .
\end{aligned}
$$

And the differential equation of dynamic density for an on-ramp (see (5)) can be written as

$$
\dot{\rho}_{\text {ramp }}(k, i)=\frac{1}{w}\left[q_{\text {rampin }}(k)-r(k, i)\right] .
$$




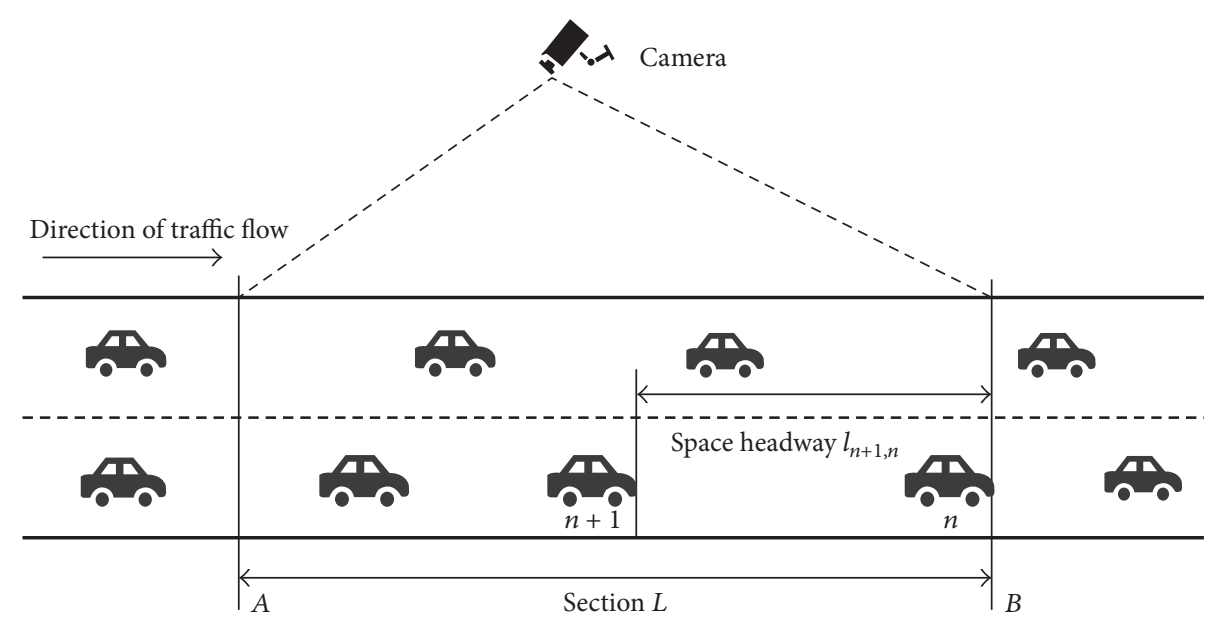

FIGURE 2: Layout scheme of data collection.

In consideration of the fact that there is an obvious difference in the usage of inner/outer lanes [33,34], the error function $J(k)$ is introduced in the MDB-RM model to adjust this difference while keeping the mainstream density close to the expected value like ALINEA and lowering the queue length at the same time. Hence,

$$
J(k)=\sum_{l=1}^{X} \lambda(l)\left|\rho(k, i, l)-\rho_{c}(i, l)\right|+\lambda_{\text {ramp }} \rho_{\text {ramp }}(k),
$$

where $\rho_{c}(i, l)$ is the expected density of lane $l$ in segment $i, \rho_{\text {ramp }}(k)$ is the density of on-ramp at time $k T, \lambda(l)$ is the weight function of lane $l, \lambda_{\text {ramp }}$ is the weight function of onramp, and $\sum \lambda(l)+\lambda_{\text {ramp }}=1$.

To minimize $J(k)$, homogeneous linear differential equation of first order is defined as

$$
p J(k)+\dot{J}(k)=0, \quad J(k)=J(0) e^{-p k T} .
$$

Further, the derivative $\dot{J}(k)$ of error function $J(k)$ can be written as

$$
\dot{J}(k)=\sum_{l=1}^{X} \lambda(l) \cdot \dot{\rho}(k, i, l)+\lambda_{\text {ramp }} \dot{\rho}_{\text {ramp }}(k) .
$$

Overall, the MDB-RM model can be written as

$$
\begin{aligned}
& r(k, i)=\frac{L \cdot w}{\lambda(X) \cdot w-\lambda_{\text {ramp }} \cdot L}\left\{\dot{J}(k)-\frac{1}{L} \sum_{l=1}^{X} \lambda(l)\right. \\
& \cdot[q(k, i-1, l)-q(k, i, l)]-\frac{1}{L} \sum_{l=1}^{X-1}[\lambda(l)-\lambda(l+1)] \\
& \cdot\left[\eta_{l+1, l}(k, i) \cdot \rho(k, i, l+1)-\eta_{l, l+1}(k, i) \cdot \rho(k, i, l)\right] \\
& \left.-\frac{\lambda_{\text {ramp }} \cdot q_{\text {rampin }}(k)}{w}\right\} .
\end{aligned}
$$

\section{Data Spectrum}

3.1. Data Collection. Validation of the proposed MDB-RM model is conducted by simulator VISSIM 7. The data for simulation is collected from a section of the Second Ring Elevated Expressway in Chengdu, a large city in southwest China. Video recording is applied for data acquisition. A HD camera was set up in the BRT station of Taoxi Road, and the recording work lasted from 17:00 to 18:40 on May 2, 2016. The layout scheme of data collection is shown in Figure 2.

$\mathrm{Wu}$ discusses how to extract traffic flow characters from traffic video recordings (e.g., "speed-density" and "speedspace headway") in his series of field studies [35-37]. Based on Wu's idea, we draw "section travel time-time headway" data directly from the video record in the first place and then obtain the vehicle speed data according to section travel time and set section length $L$.

Define $A$ as origin and $B$ as destination of the road section. In the data extraction process, the expressway is simplified as a one-way road with two lanes. Assume that $t_{n}^{A}$ is the time when vehicle $n$ arrived at $A$ and $t_{n}^{B}$ is the time when $n$ arrived at $B$. Then, the time when the next vehicle $n+1$ arrived at $B$ is $t_{n+1}^{B}$. Thus, the section travel time of vehicle $n$ can be represented as $t_{n}^{V}=t_{n}^{B}-t_{n}^{A}$. The time headway of vehicle $n+1$ can be represented as $t_{n+1}^{D}=t_{n+1}^{B}-t_{n}^{B}$.

Further, the section speed $v_{n}$ can be written as

$$
v_{n}=\frac{L}{t_{n}^{V}}=\frac{L}{t_{n}^{B}-t_{n}^{A}} .
$$

When the head of vehicle $n$ arrives at $B$, the location of vehicle $n+1$ in the same lane can be shown in Figure 2. Define the space headway between vehicle $n$ and vehicle $n+1$ as $l_{n+1, n}$. Thus, the space headway $l_{n+1, n}$ can be approximately represented as the product of the section speed $v_{n+1}$ and the time headway $t_{n+1}^{D}$, shown as

$$
l_{n+1}=v_{n+1} \cdot t_{n+1}^{D}=v_{n+1} \cdot\left(t_{n+1}^{B}-t_{n}^{B}\right) .
$$


According to the relationship between space headway and density, the density of vehicle $n+1$ can be represented as

$$
\rho_{n+1}=\frac{1000}{l_{n+1}} .
$$

Although time counting of the recordings is operated manually, the video playback can be slowed down to the level of a frame (the video recording lasts 100 minutes which is equal to 150000 frames). The error of section travel time recording is controlled in 3 frames. That is, for a vehicle traveling at speed of $50 \mathrm{~km} / \mathrm{h}$, the relative error of speed is less than $6.329 \%$, which is acceptable for our research purpose. Moreover, since the data is collected during the peak hour of daily traffic, most vehicles were traveling at a relatively low speed. The data processing in level of a frame ensures the accuracy of the traffic flow data.

3.2. Data Description. After data extraction, two principles are applied for data filtering: first, the section speed of the vehicle cannot be higher than $100 \mathrm{~km} / \mathrm{h}(80 \mathrm{~km} / \mathrm{h}$ is the speed limit on the Second Ring Elevated Expressway); second, density cannot be greater than $150 \mathrm{veh} / \mathrm{km}$. Finally, 3385 pairs of "section travel time-time headway" and "section speeddensity" are obtained.

To illustrate the traffic features of the Second Ring Elevated Expressway, time-varying diagrams of traffic volume, speed, and density are separately plotted, shown as Figure 3. The traffic volume data is obtained on the basis of the rate of flow for 20-second intervals instead of the flow-speed-density formula. The number of passing vehicles is counted over every 20 seconds to reflect short-term fluctuations of traffic in as much detail as possible $[38,39]$. As seen in Figure 3, operation features of the Second Ring Elevated Expressway during the peak hour of the observed day can be concluded as follows:

(i) In the first 30 minutes during the data collection, the traffic is mostly under free flow condition. Thus, the traffic flow fluctuation is relatively large while the speed and density are stable. The traffic jams in this time period are nonrecurrent and temporary, such as the breakdowns observed in the 21st-23rd minute and the 25-27th minute.

(ii) Viewed from the speed time-varying diagram, vehicle speed has a significant drop from the 39th minute, which implies the start of recurrent congestion. It is also observed that the congestion lasts from the 41st to the $63 \mathrm{rd}$ minute. Most vehicles travel at $10-20 \mathrm{~km} / \mathrm{h}$, and density during this time internal is relatively high. As for traffic volume, though the fluctuation decreases, the 20 -second rate of flow in some intervals decreases since merging vehicles increase the congestion in the mainstream (such as the flow rate of 0 at the 43rd minute).

(iii) Evacuation of vehicles in the mainstream starts from the 64 th minute, and the speed rises back to $60 \mathrm{~km} / \mathrm{h}$ in a very short time. The main reason is the decrease of both vehicle arrival and downstream traffic flow (there is an obvious trough during the 64-67th minute in time-varying diagram of flow rate). Due to the rebound of vehicles, the speed drops again during the 69-85th minute, as shown in speed time-varying diagram.

(iv) The traffic recovers to free flow after the 85th minute. More randomness of vehicle arrival, which is shown as the large vibration in flow rate time-varying diagram, can be seen during this time period. The speed of vehicles is generally higher than the average, and the density descends to the same level with the first 30 minutes.

While Figure 3 reveals traffic flow features of the investigated road, on the other hand, the consistency of the traffic variation tendency indicated by the volume-speed-density data also proves the reliability of the collected data. Moreover, we translate the "section speed-density" data into "traffic volume-density" data and plot the scatter diagram to represent the relationship between these traffic flow parameters in Figure 4. In this way, the effectiveness of our field data is further supported.

Taking density variation in multilane situations into account, the error of traffic volume in the whole road cannot be neglected. Thus, vacancy parameter $\lambda$ is introduced to fix volume $Q^{\prime}$ as follows:

$$
Q^{\prime}=\lambda \bar{v}_{s} \cdot \rho=\lambda \bar{v}_{s} \frac{1000}{h} .
$$

The value of $\lambda$ is related to both the level of congestion in real situations and the counted number of vehicles. In this paper, we put $\lambda=0.77$ based on former experience.

Figure 4 shows the relationship between speed and density as well as volume and density in terms of a scatter diagram. Regression analysis is conducted for the "speeddensity" data using the Greenshields, Underwood, and Greenburg models separately. The result indicates that all the models are statistically significant and that the Underwood model fits best, which is identical to another research outcome in Beijing [40]. The basic trend of the "volumedensity" diagram can also be supported by Kerner and Rehborn's conclusion in 1996 [41]. In his empirical study with large amounts of field data, Kerner points out that, under low density situation (free flow condition), the "volumedensity" can be simplified as a curve and a two-dimensional area under middle/high density situation (congestion). It also explains why the linearity is not very significant in Figure 4(b), since free flow condition in the investigated road rarely occurs during the peak hour.

\section{Simulation and Discussion}

4.1. Simulation. The simulation is conducted for two monodirectional, homogeneous lanes of the freeway mainstream with a single on-ramp. The interval distance $L$ between the mainstream detectors and the interval distance $w$ between on-ramp detectors are set equal to 0.5 and $0.3 \mathrm{~km}$, respectively. The time interval $T$ is set equal to $30 \mathrm{~s}$ for all control scenarios. Four evaluation scenarios 

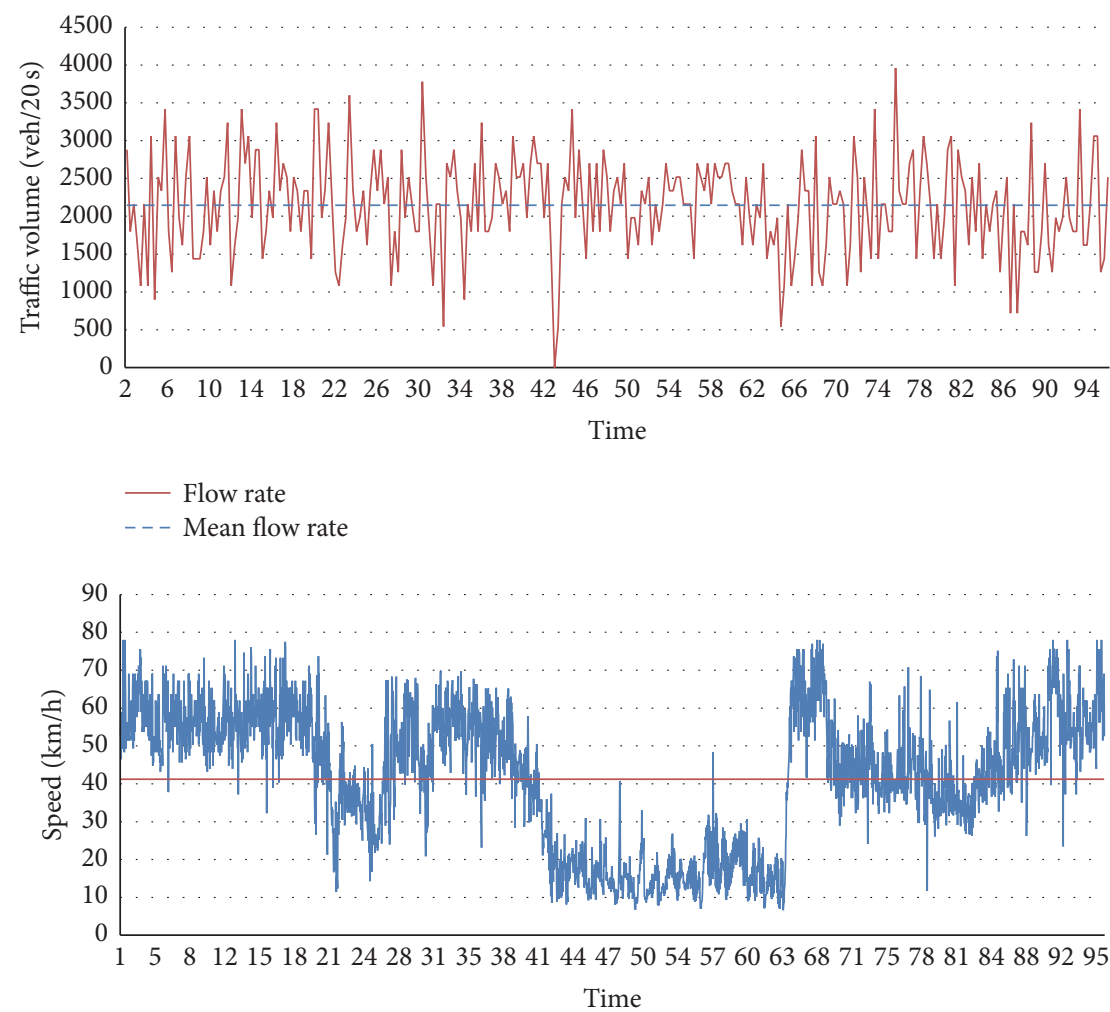

- Speed

- Mean speed

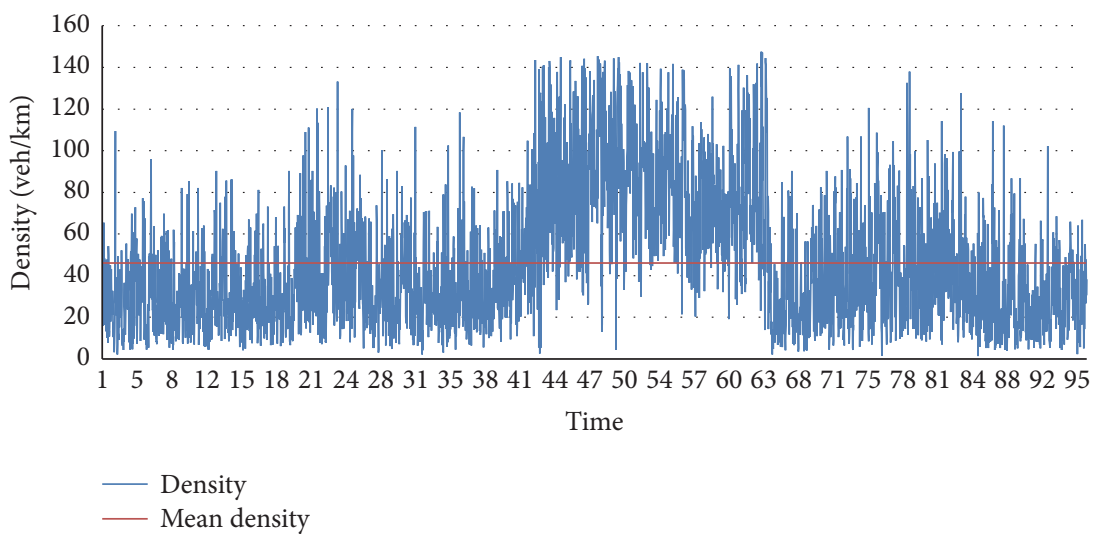

FIGURE 3: Time-varying diagram of traffic volume, speed, and density.

are tested, that is, noncontrol, ALINEA, DB-RM (densitybased ramp metering model), and MDB-RM. The ALINEA algorithm implemented is the ALINEA module based on VisVAP. VISSIM COM (Component Object Model) of API (Application Programming Interface) is used to implement the MDB-RM and DB-RM control scenarios. An optimal occupancy threshold 0.3 is calibrated to achieve the optimal performance of ALINEA.

The field data presented in Section 3 is converted into traffic counts as inputs for the simulator. The traffic flows of the mainstream and on-ramp are set as $2185 \mathrm{veh} / \mathrm{h}$ and $620 \mathrm{veh} / \mathrm{h}$, respectively. The expected densities of lane 1 and lane 2 are separately set as $\rho_{c}(i, 1)=63 \mathrm{veh} / \mathrm{km}$ and $\rho_{c}(i, 2)=$ $69 \mathrm{veh} / \mathrm{km}$. Moreover, define $\lambda(1)=0.4, \lambda(2)=0.45, \lambda_{\text {ramp }}=$ 0.15 , and $k=0.95$.

We run the simulation with five random seeds, and the time length of each simulation investigation is 1 hour. The results are evaluated by comparing the mean speed of the mainstream, mean travel time of the mainstream and onramp, mean vehicle delay of the mainstream and on-ramp, and the mean queue length of the on-ramp. Table 1 shows the main output data of simulation.

To further analyze the key evaluation indexes, the mean travel times of the mainstream and on-ramp are counted 


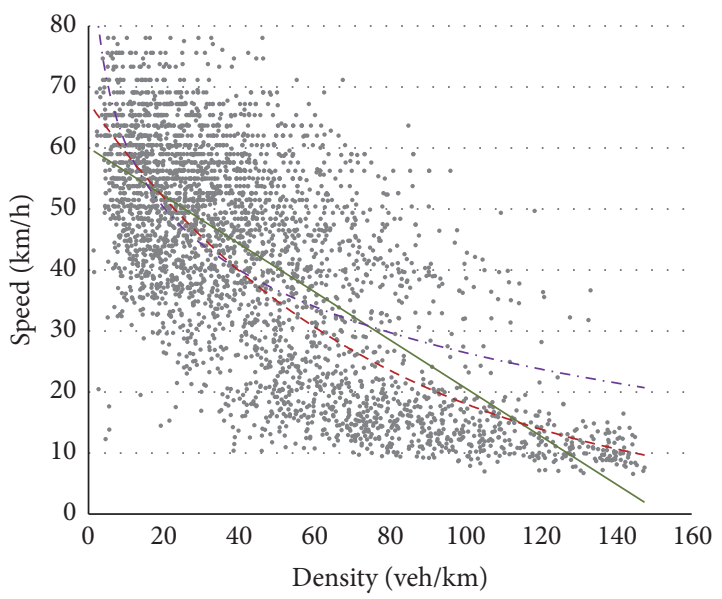

$\begin{array}{ll}\text { Measured value } & -\cdot-\text { Greenburg } \\ \text { Greenshields } & \text { - - - Underwood }\end{array}$

(a) "Speed-density" scatter diagram

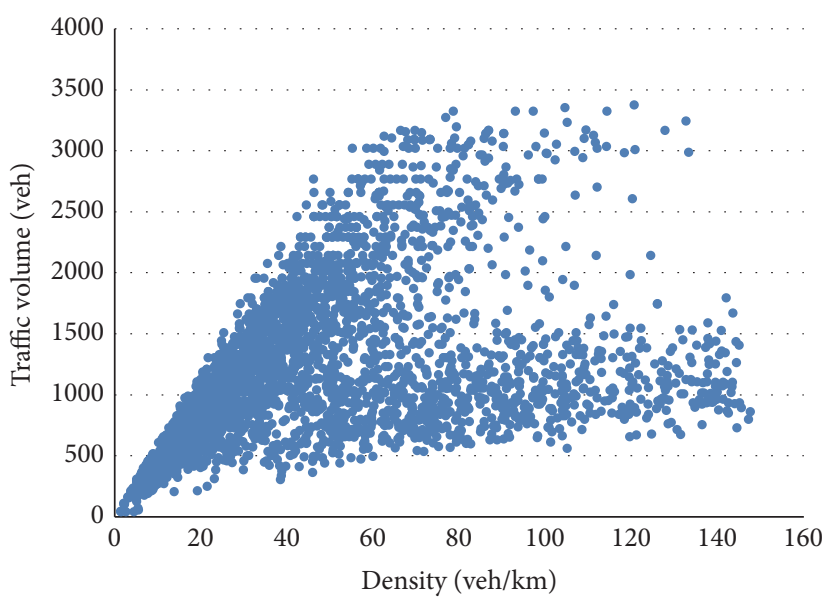

(b) "Traffic volume-density" scatter diagram

FiguRE 4: Scatter diagram of traffic flow parameter.
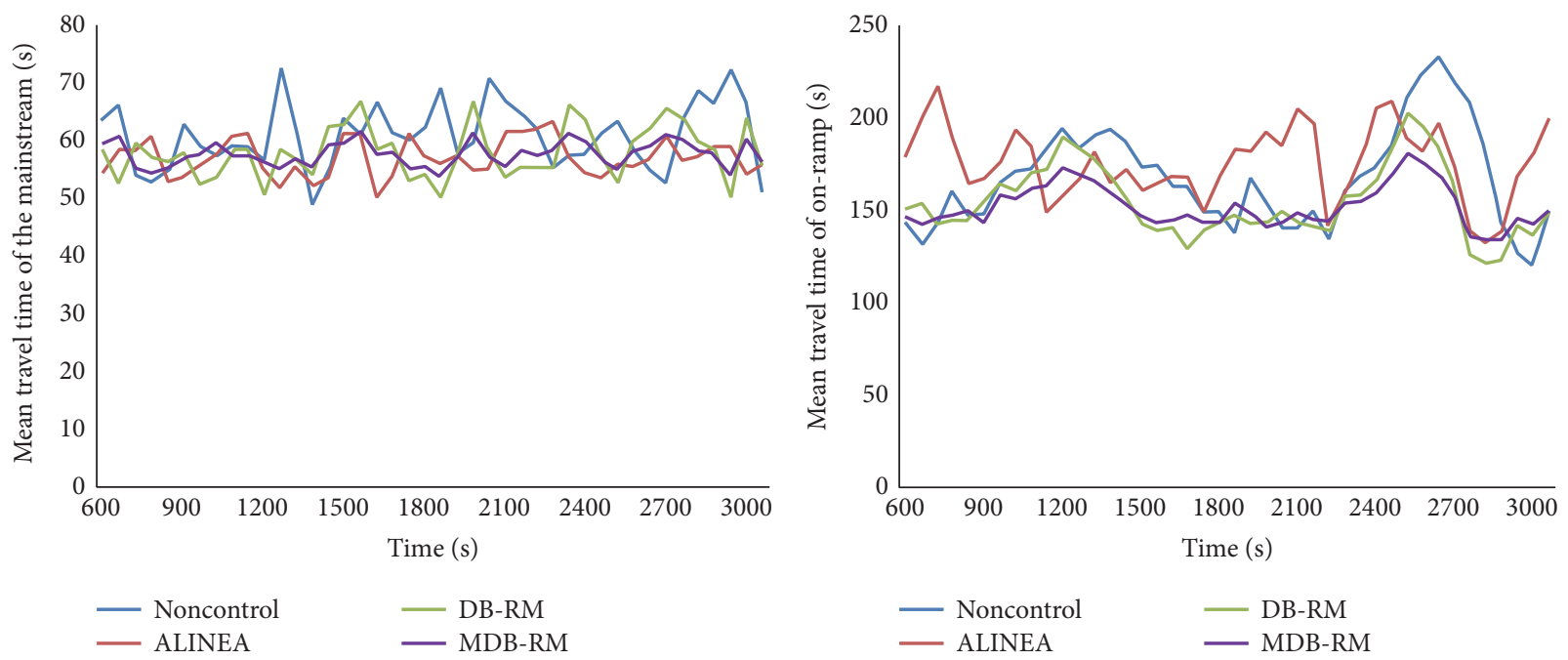

FIgURE 5: Mean travel time of the mainstream and on-ramp.

from the 600th to the 3000th second in every 60 seconds. 52 samples are obtained from the simulator for each control scenario. The variation trends are drawn in Figure 5.

Moreover, in order to prove the advantages of MDB-RM model in population, $Z$-test is conducted for mean travel time of the mainstream and on-ramp. $Z$-statistic yields

$$
Z=\frac{\left(\bar{X}_{1}-\bar{X}_{2}\right)-\left(\mu_{1}-\mu_{2}\right)}{\sqrt{S_{1}^{2} / n_{1}+S_{2}^{2} / n_{2}}},
$$

where $\bar{X}_{i}$ represents the mean value of sample $i . \mu_{i}$ represents the mean value of population $i$ (in null hypothesis: $\mu_{1}=\mu_{2}$ ). $S_{i}{ }^{2}$ represents variance of sample $i$. Since the sample sizes of the above four simulation schemes are all equal to 52 , mean travel time of vehicles in the mainstream and onramp can be approximately regarded as normal distribution. Compare the output results of MDB-RM model with those from noncontrol, ALINEA, and DB-RM model, respectively. Assume that using MDB-RM model can statistically reduce mean travel time of vehicles both in the mainstream and in on-ramp. In the 95 percent confidence interval, the critical value of $Z$-test is equal to 1.645 . The relevant parameters and results of the $Z$-test are shown in Table 2.

As seen from Table 2, for mean travel time of on-ramp, the $Z$-statistics are all smaller than the critical $Z$-value (i.e., 1.645). Thus, the null hypothesis cannot be rejected, which indicates that mean travel time of on-ramp is significantly decreased when using the MDB-RM model. As for the results in the mainstream, we can also conclude that the MDB-RM model has a significant advantage over other models, except 
TABLE 1: Main output data of simulation.

\begin{tabular}{|c|c|c|c|c|c|c|c|}
\hline \multirow{2}{*}{ Index } & \multirow{2}{*}{ Noncontrol } & \multicolumn{2}{|c|}{ ALINEA } & \multicolumn{2}{|c|}{ DB-RM } & \multicolumn{2}{|c|}{ MDB-RM } \\
\hline & & Mean output & Change rate (\%) & Mean output & Change rate (\%) & Mean output & Change rate (\%) \\
\hline $\begin{array}{l}\text { Mean speed of the } \\
\text { mainstream } \\
(\mathrm{km} / \mathrm{h})\end{array}$ & 30.6 & 33.5 & 9.48 & 32.9 & 7.52 & 33.1 & 8.17 \\
\hline $\begin{array}{l}\text { Mean travel time of } \\
\text { the mainstream (s) }\end{array}$ & 60.5 & 56.7 & -6.28 & 57.3 & -5.29 & 57.9 & -4.30 \\
\hline $\begin{array}{l}\text { Mean travel time of } \\
\text { on-ramp (s) }\end{array}$ & 167.3 & 173.2 & 3.53 & 154.2 & -7.83 & 152.5 & -8.85 \\
\hline $\begin{array}{l}\text { Mean vehicle delay } \\
\text { of the mainstream } \\
\text { (s) }\end{array}$ & 24.7 & 22.5 & -8.91 & 23.1 & -6.48 & 22.9 & -7.29 \\
\hline $\begin{array}{l}\text { Mean vehicle delay } \\
\text { of on-ramp (s) }\end{array}$ & 85.4 & 95.6 & 11.94 & 81.2 & -4.92 & 80.5 & -5.74 \\
\hline $\begin{array}{l}\text { Mean queue length } \\
\text { of on-ramp }(m)\end{array}$ & 107.3 & 117.5 & 9.51 & 93.5 & -12.86 & 92.9 & -13.42 \\
\hline
\end{tabular}

TABLE 2: Relevant parameters and results of the $Z$-test.

\begin{tabular}{|c|c|c|c|c|c|}
\hline Evaluation index & Parameter & Noncontrol & ALINEA & DB-RM & MDB-RM \\
\hline \multirow{4}{*}{$\begin{array}{l}\text { Mean travel time of } \\
\text { the mainstream }\end{array}$} & Mean $\left(\bar{X}_{i}\right)$ & 60.5 & 56.7 & 57.3 & 57.9 \\
\hline & Variance $\left(S_{i}^{2}\right)$ & $5.29^{2}$ & $3.16^{2}$ & $4.39^{2}$ & $2.12^{2}$ \\
\hline & Sample size $n_{i}$ & 52 & 52 & 52 & 52 \\
\hline & $Z$-statistic & -3.40 & 2.21 & 0.83 & - \\
\hline \multirow{4}{*}{$\begin{array}{l}\text { Mean travel time of } \\
\text { on-ramp }\end{array}$} & Mean $\left(\bar{X}_{i}\right)$ & 167.3 & 173.2 & 154.2 & 152.5 \\
\hline & Variance $\left(S_{i}^{2}\right)$ & $25.56^{2}$ & $19.56^{2}$ & $18.64^{2}$ & $10.91^{2}$ \\
\hline & Sample size $n_{i}$ & 52 & 52 & 52 & 52 \\
\hline & $Z$-statistic & -3.83 & -6.68 & -0.58 & - \\
\hline
\end{tabular}

for ALINEA. This is not a surprise since our primary objective of modeling is to improve the efficiency of on-ramp control.

4.2. Discussion. Generally speaking, all these three control methods (ALINEA, DB-RM, and MDB-RM) are capable of increasing the mainstream speed and reducing the mainstream travel time. Yet, ALINEA best performs in terms of increasing the mainstream speed and saving time. On the other hand, compared with ALINEA and DB-RM, the MDBRM model displays significant improvements in the aspects of mean travel time, mean vehicle delay, and mean queue length of the on-ramp. Also, Figure 5 suggests that the curves of mean travel time in both the mainstream and the on-ramp are the smoothest when using the MDB-RM model. This is mainly because the design principle of the MDB-RM model is concerned with not only the on-ramp density, but also the difference of multilane density in the mainstream, something which is absent in the DB-RM model. Meanwhile, the onramp queue length has not been taken into consideration in ALINEA.

The main objective of the MDB-RM model is to keep the lane density around the expected value. This explains why the MDB-RM model reaches a similar achievement to the ALINEA and DB-RM. In total, MDB-RM, DB-RM, and ALINEA improve the mean speed of the mainstream by $8.17 \%, 7.52 \%$, and $9.48 \%$, respectively. Additionally, the mean travel time of the mainstream decreases by $5.29 \%$, $4.3 \%$, and $6.28 \%$ separately in each scenario. The reason why less improvement in the mainstream is observed when using the MDB-RM and DB-RM models is mainly caused by the synchronous consideration of both the mainstream flow and the on-ramp queue. Moreover, the gaps between their optimal results are relatively small.

However, observing from the on-ramp side, the improvements achieved by the MDB-RM model are noteworthy. Mean travel time of the on-ramp is reduced by $8.67 \%$. Additionally, there is a $7.29 \%$ drop in mean vehicle delay in the mainstream. A $5.57 \%$ and a $12.42 \%$ decrease of mean vehicle delay and mean queue length of the on-ramp, respectively, are also obtained when using the MDB-RM model. By contrast, using ALINEA will increase by $3.53 \%$ the on-ramp mean travel time, along with an $11.94 \%$ increase of mean vehicle delay and a 9.51\% increase for mean queue length of on-ramp.

Overall, the simulation results demonstrate that the MDB-RM model outperforms the DB-RM model in every evaluation index and they also reach almost the same 
effectiveness for improving the mainstream traffic conditions compared with the most well-known algorithm, ALINEA, while behaving much better than ALINEA in the on-ramp section. Thus, we can conclude that the proposed model is capable of achieving a comprehensive optimal result from both sides of the mainstream and on-ramp.

\section{Conclusion}

In this paper, an MDB-RM model aimed at realizing the same control effect as ALINEA as well as improving on-ramp traffic condition is developed. Dynamic density is chosen as the optimal index due to its convenient accessibility in practical area. The error function is introduced to adjust the density difference between lanes. In this way, lane change behavior is included in the multilane dynamic density-based model. With the field traffic flow data collected in Chengdu, simulation for the MDB-RM model is conducted on the platform of VISSIM COM and VisVAP, along with three other scenarios, that is, noncontrol, ALINEA, and DB-RM. The simulation result shows that the MRD-RM model is capable of reducing the queue length of on-ramp while keeping the traffic volume of the mainstream close to capacity.

Furthermore, the simulation results indicate that the MRD-RM model outperforms ALINEA with 11-22\% in the evaluation indexes for the on-ramp, while comprehensively surpassing the DB-RM model. The simulation results also illustrate the potential of using MRD-RM as an effective alternative in addressing congestion caused by merging traffic.

On the other hand, when the traffic demands of both the mainstream and the on-ramp grow largely, especially when capacity is exceeded, there will be a tradeoff between reducing ramp queue and maximizing the mainstream traffic flow. How to balance these two sides and reach system optimization requires further discussion.

\section{Competing Interests}

The authors declare that there are no competing interests regarding the publication of this paper.

\section{Acknowledgments}

This study is supported by the Sichuan Provincial Scientific Research Innovation Team Program (17TD0035), Sichuan Provincial Science and Technology Program (2017ZR0032), Chengdu Science and Technology Project (2015-RK0000227-ZF), 2015 Natural Science Key Foundation of Xihua University (no. Z1520315), and the Open Research Subject of Key Laboratory of Vehicle Measurement, Control and Safety, Xihua University (szjj2016-014, szjj2015-044).

\section{References}

[1] M.-H. Ma, Q.-F. Yang, S.-D. Liang, and Z.-L. Li, "Integrated variable speed limits control and ramp metering for bottleneck regions on freeway," Mathematical Problems in Engineering, vol. 2015, Article ID 313089, 17 pages, 2015.

[2] G. N. Bifulco, G. E. Cantarella, F. Simonelli, and P. Velonà, "Advanced traveller information systems under recurrent traffic conditions: network equilibrium and stability," Transportation Research Part B: Methodological, vol. 92, pp. 73-87, 2016.

[3] M. Papageorgiou and A. Kotsialos, "Freeway ramp metering: an overview," IEEE Transactions on Intelligent Transportation Systems, vol. 3, no. 4, pp. 271-281, 2002.

[4] M. Papageorgiou, E. Kosmatopoulos, I. Papamichail, and Y. Wang, "ALINEA maximises motorway throughput-an answer to flawed criticism," Traffic Engineering and Control, vol. 48, no. 6, pp. 271-276, 2007.

[5] J. Fang, "Performance tuning of coordinated active traffic control algorithm: simultaneously improving corridor safety and mobility performances," Mathematical Problems in Engineering, vol. 2014, Article ID 130804, 11 pages, 2014.

[6] California Department of Transportation, 2013 Ramp Metering Annual Report, http://www.dot.ca.gov/dist07/resources/ramp_ metering/docs/2013_RMAR_Nov2014.pdf.

[7] H. Hadj-Salem, J. M. Blosseville, and M. Papageorgiou, "ALINEA. A local feedback control law for on-ramp metering; a real-life study," in Proceedings of the 3rd International Conference on Road Traffic Control, pp. 194-198, London, UK, May 1990.

[8] H. Hadj-Salem, M. M. Davée, and J. M. Blosseville, "ALINEA: un outil de regulation d'accés isolé sur autoroute," Report INRETS 80, Institut de Recherche des Transports, Arcueil, France, 1988.

[9] S. Smulders and F. Middelham, Isolated Ramp Metering: RealLife Study in the Netherlands, Deliverable 7a, Project CHRISTIANE (V1035), DRIVE Office, Brussels, Belgium, 1991.

[10] M. Papageorgiou, J.-M. Blosseville, and H. Haj-Salem, "Modelling and real-time control of traffic flow on the southern part of boulevard peripherique in Paris: part II: coordinated onramp metering," Transportation Research Part A: General, vol. 24, no. 5, pp. 361-370, 1990.

[11] L. Zhang and D. Levinson, "Optimal freeway ramp control without origin-destination information," Transportation Research Part B: Methodological, vol. 38, no. 10, pp. 869-887, 2004.

[12] L. Lipp, L. Corcoran, and G. Hickman, "Benefits of central computer control for the denver ramp metering system," Transportation Research Record, vol. 1320, pp. 3-6, 1990.

[13] H. Haj-Salem, N. Farhi, and J. P. Lebacque, "Field evaluation results of new isolated and coordinated ramp metering strategies in France," IFAC Proceedings Volumes, vol. 45, no. 6, pp. 378-383, 2012.

[14] L. Faulkner, F. Dekker, D. Gyles, L. Papamichail, and M. Papageorgiou, "Evaluation of HERO-Coordinated ramp metering installation at M1 and M3 freeways in Queensland, Australia," Transportation Research Record, vol. 2470, pp. 13-23, 2014.

[15] A. Alessandri, A. Di Febbraro, A. Ferrara, and E. Punta, "Optimal control of freeways via speed signalling and ramp metering," Control Engineering Practice, vol. 6, no. 6, pp. 771780, 1998.

[16] M. Papageorgiou, H. Hadj-Salem, and F. Middelham, "ALINEA local ramp metering: summary of field results," Transportation Research Record, no. 1603, pp. 90-98, 1997.

[17] P. Masher, D. W. Ross, and P. J. Wong, Guidelines for Design and Operation of Ramp Control Systems, Stanford Research Institute, 1975.

[18] H. M. Koble and V. S. Samant, Control Strategies in Response to Freeway Incidents, Federal Highway Administration, Offices of Research \& Development, Traffic Systems Division, 1980.

[19] X. Yang, L. Chu, and W. Recker, "GA-based parameter optimization for the ALINEA ramp metering control," in Proceedings 
of the 5th IEEE International Conference on Intelligent Transportation Systems (ITSC '02), pp. 627-632, IEEE, Singapore, September 2002.

[20] L. Chu and X. Yang, "Optimization of the ALINEA rampmetering control using genetic algorithm with microsimulation," in Proceedings of the Transportation Research Board Annual Meeting, vol. 37, pp. 2918-2924, 2003.

[21] E. Smaragdis and M. Papageorgiou, "Series of new local ramp metering strategies," Transportation Research Record, no. 1856, pp. 74-86, 2003.

[22] E. Smaragdis, M. Papageorgiou, and E. Kosmatopoulos, "A flowmaximizing adaptive local ramp metering strategy," Transportation Research Part B: Methodological, vol. 38, no. 3, pp. 251-270, 2004.

[23] Y. Wang and M. Papageorgiou, "Local ramp metering in the case of distant downstream bottlenecks," in Proceedings of the IEEE Intelligent Transportation Systems Conference (ITSC '06), September 2006.

[24] Y. Wang, M. Papageorgiou, J. Gaffney, I. Papamichail, and J. Guo, "Local ramp metering in the presence of random-location bottlenecks downstream of a metered on-ramp," in Proceedings of the 13th International IEEE Conference on Intelligent Transportation Systems (ITSC '10), vol. 2178, pp. 1462-1467, Madeira Island, Portugal, September 2010.

[25] R. Chi, Z. Hou, S. Jin, D. Wang, and J. Hao, "A data-driven iterative feedback tuning approach of ALINEA for freeway traffic ramp metering with PARAMICS simulations," IEEE Transactions on Industrial Informatics, vol. 9, no. 4, pp. 23102317, 2013.

[26] S. Jin, Z. Hou, R. Chi, and J. Hao, "A data-driven control design approach for freeway traffic ramp metering with virtual reference feedback tuning," Mathematical Problems in Engineering, vol. 2014, Article ID 936531, 8 pages, 2014.

[27] W.-B. Gao, J. Wu, and J. Zou, "On-ramp metering algorithm based on real-time density," Journal of Transportation Systems Engineering and Information Technology, vol. 12, no. 2, pp. 150155, 2012.

[28] M. J. Lighthill and G. B. Whitham, "On kinematic waves. II. A theory of traffic flow on long crowded roads," Proceedings of the Royal Society: Mathematical, Physical and Engineering Sciences, vol. 229, no. 1178, pp. 317-345, 1955.

[29] M. Papageorgiou, B. Posch, and G. Schmidt, "Comparison of macroscopic models for control of freeway traffic," Transportation Research Part B, vol. 17, no. 2, pp. 107-116, 1983.

[30] M. Papageorgiou, J.-M. Blosseville, and H. Hadj-Salem, "Macroscopic modelling of traffic flow on the Boulevard Périphérique in Paris," Transportation Research Part B, vol. 23, no. 1, pp. 29-47, 1989.

[31] A. Messmer and M. Papageorgiou, "METANET: a macroscopic simulation program for motorway networks," Traffic Engineering \& Control, vol. 31, no. 9, pp. 466-470, 1990.

[32] A. Kotsialos, M. Papageorgiou, C. Diakaki, Y. Pavlis, and F. Middelham, "Traffic flow modeling of large-scale motorway networks using the macroscopic modeling tool METANET," IEEE Transactions on Intelligent Transportation Systems, vol. 3, no. 4, pp. 282-292, 2002.

[33] Z.-X. Lin, Z. Wu, C.-H. Yang, and X.-Q. Zheng, "A study on traffic flow models based on measure video of Yanan Expressway of Shanghai," Chinese Journal of Hydrodynamics, vol. 25, no. 5, pp. 683-693, 2010.

[34] Z. Wu, M. Guo, X. Zheng et al., "Measured data analysis of urban Expressway and research on traffic flow models," Chinese
Journal of Theoretical and Applied Mechanics, vol. 42, no. 4, pp. 789-797, 2010.

[35] Z. Wu, H. Zhu, and N. Jia, "Measuring method study of the traffic flow model parameters based on video recording of expressway traffic," Journal of Fudan University (Nature Science), vol. 47, no. 2, pp. 147-152, 2008.

[36] Z. Lin, Z. Wu, and C. Yang, "A study on traffic flow models based on measure video of yanan expressway of Shanghai," Chinese Journal of Hydrodynamics, vol. 25, no. 5, pp. 683-693, 2010.

[37] Z. Wu, M. Guo, X. Zheng et al., "Measured data analysis of urban expressway and research on traffic flow models," Chinese Journal of Theoretical and Applied Mechanics, vol. 42, no. 4, pp. 789-797, 2010.

[38] M. J. Cassidy and R. L. Bertini, "Some traffic features at freeway bottlenecks," Transportation Research Part B: Methodological, vol. 33, no. 1, pp. 25-42, 1999.

[39] R. L. Bertini and M. T. Leal, "Empirical study of traffic features at a freeway lane drop," Journal of Transportation Engineering, vol. 131, no. 6, pp. 397-407, 2005.

[40] X. Sun, H. Lu, and J. Wu, "Research on speed-flow-density relationship model of Beijing urban expressway," Highway Engineering, vol. 37, no. 1, pp. 43-48, 2012.

[41] B. S. Kerner and H. Rehborn, "Experimental properties of complexity in traffic flow," Physical Review E, vol. 53, no. 5, pp. 4275-4278, 1996. 


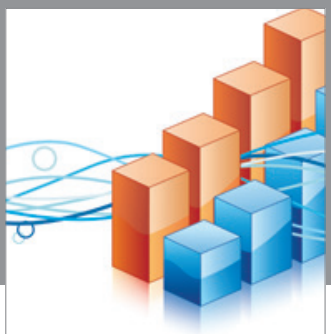

Advances in

Operations Research

vatem alat4

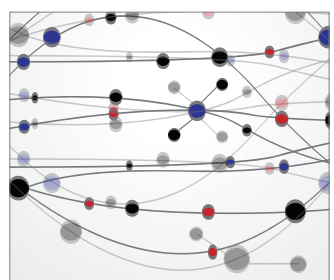

\section{The Scientific} World Journal
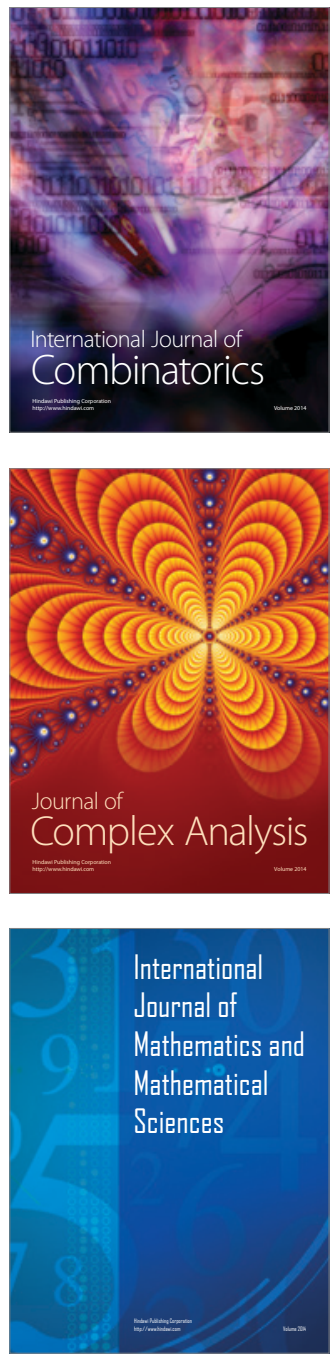
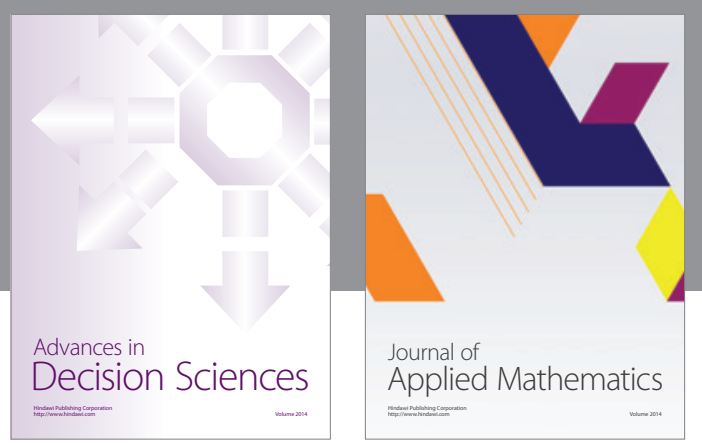

Algebra

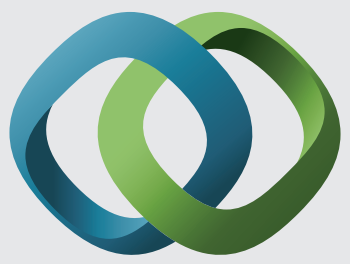

\section{Hindawi}

Submit your manuscripts at

https://www.hindawi.com
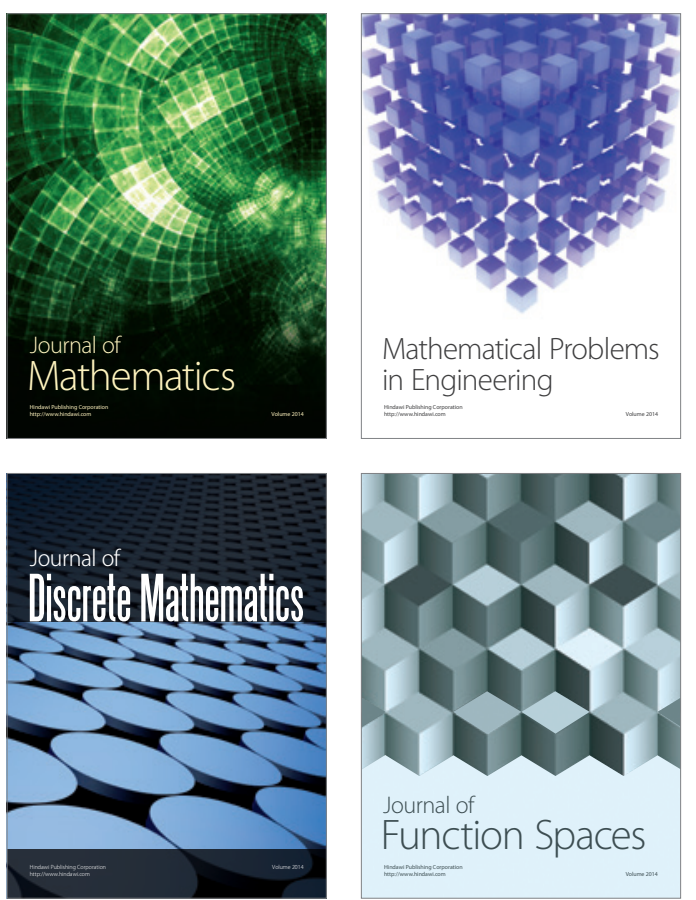

Mathematical Problems in Engineering
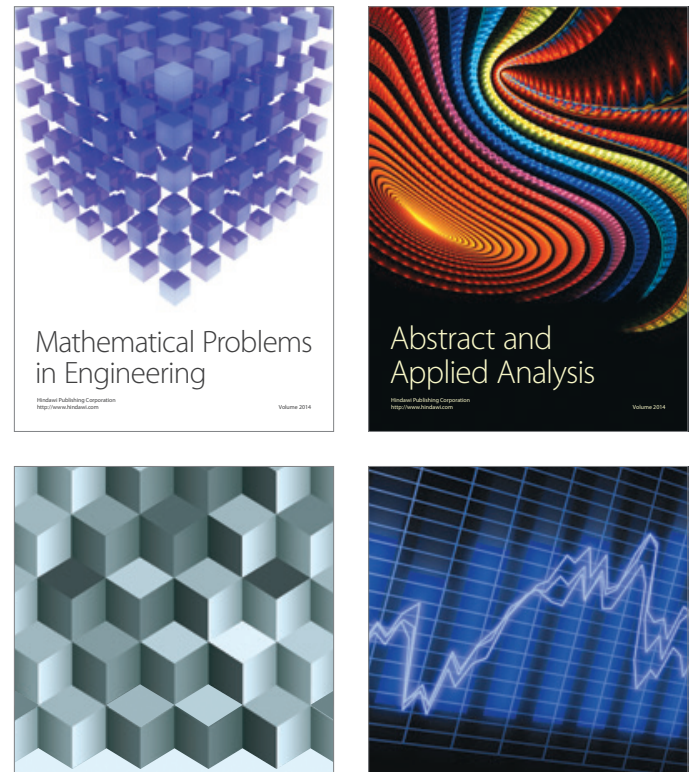

Journal of

Function Spaces

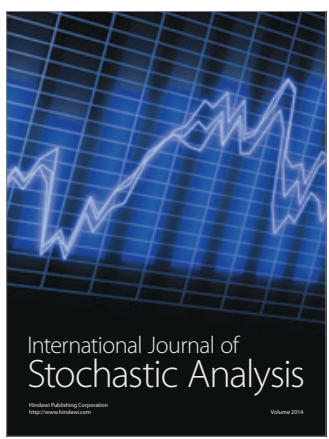

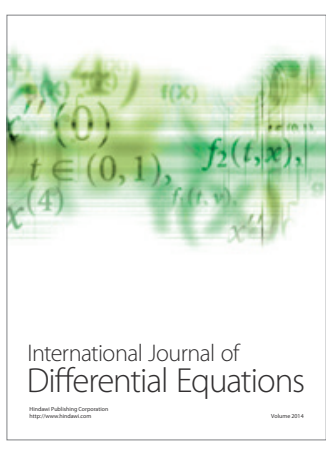
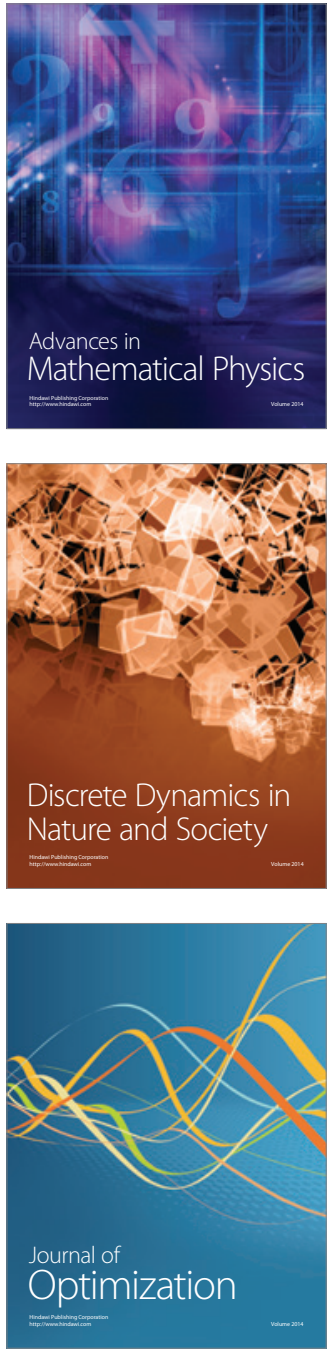